\title{
Naturally occurring graphite cones
}

\author{
John A. Jaszczak ${ }^{\mathrm{a}, *}$, George W. Robinson ${ }^{\mathrm{b}}$, Svetlana Dimovski ${ }^{\mathrm{c}}$, and Yury Gogotsi ${ }^{\mathrm{c}}$ \\ ${ }^{a}$ Department of Physics and the A. E. Seaman Mineral Museum, Michigan Technological \\ University, Houghton, Michigan, 49931-1295, USA \\ ${ }^{b}$ Department of Geological and Mining Engineering and Sciences, and the A. E. Seaman \\ Mineral Museum, Michigan Technological University, Houghton, Michigan, 49931-1295, \\ USA \\ ${ }^{c}$ Department of Materials Science and Engineering, Drexel University, 3141 Chestnut St., \\ Philadelphia, PA 19104, USA \\ *Corresponding author. Tel. +1-906-487-2255; fax: +1-906-487-2933. \\ E-mail address: jaszczak@mtu.edu (J. A. Jaszczak)
}

\begin{abstract}
Carbon, boron nitride, and other materials that form nanotubes are also able to form conical shapes. Even though potential applications of cone arrays as electron emitters and other devices are very promising, understanding of their structure and formation mechanisms is still very limited compared to nanotubes and other carbon structures. Moreover, the cones have only been synthesized in a mixture with other shapes, but never as continuous arrays. It appears, however, that we can learn from nature how to produce large carbon cone arrays. We here report the first-known natural occurrence of large arrays of conical graphite crystals. These occur on the surfaces of millimeter-sized polycrystalline spheroidal aggregates of graphite. Cone heights range from less then a micron to $40 \mu \mathrm{m}$, which are larger than any other carbon cones reported in literature. They are also observed to dominate sample surfaces. Surface topography of the cones and petrologic relations of the samples suggest that the cones formed from a metamorphic fluid. Unlike most laboratoryproduced cones, the natural cones have a wide distribution of apex angles, which supports a disclination model for cone-helix structures.
\end{abstract}

Key Words- A. Natural graphite; B. Hydrothermal treatment; C. Scanning electron microscopy, Raman spectroscopy; D. Microstructure

Accepted for publication in Carbon (2003). 


\section{INTRODUCTION}

Graphite and structurally similar materials show an amazing versatility to form a wide variety of unusual morphologies, textures and structures from the macroscopic to the nanoscale [1-9]. This stems from graphite's crystal structure: layers of graphene sheets weakly bonded to each other in a staggered stacking sequence. Novel graphitic carbon structures continue to be of tremendous interest because of their promise for potential technological applications taking advantage of their unique mechanical and electrical properties. Both synthetic and natural graphite are known to occur as tabular and columnar hexagonal prisms, whiskers, complex networks of contorted sheets, and even spheres [10]. This variety of morphologies and microstructures results from distortions of the graphene sheets during growth and by the incorporation of various defects such as dislocations, disclinations, twin planes and pentagonal and heptagonal rings. A very interesting class of exotic graphitic materials is comprised of cone and cone-helix structures, which have been grown at the micro- and nano-scales [3-7,11-17], but always accompanied by other tubular or planar graphite structures.

\section{MATERIALS AND METHODS}

Spherical, spheroidal, and "triskelial" aggregates of graphite ( 0.1 to $10 \mathrm{~mm}$ in diameter) [18] occur in calcite boudins up to $30 \mathrm{~cm}$ across from the Bancroft shear zone $[19,20]$ in the Central Metasedimentary Belt of the Canadian Grenville province, and are exposed at roadcuts 3-5 km south of Gooderham, Ontario, Canada. Peak metamorphic temperatures in the area are believed to be below $700^{\circ} \mathrm{C}$ [19]. The graphite spheres were exposed by mechanical trimming and $\mathrm{HCl}$ dissolution of the enclosing calcite. X-ray diffraction studies of whole spheres indicate the graphite is well crystallized and hexagonal. Their surfaces have varied textures, including smooth, scaly, velvety, and crystalline. Those with velvety surfaces have a structure resembling the skeletal graphite overgrowths described by Weis [21] and some of them were covered with the graphite cones (Fig. 1). The graphite spheres are commonly circumscribed with one or more thin, protruding graphite ridges (Fig. 1a), which in thin section can often clearly be correlated with calcite grain boundaries, thus suggesting fluid deposition of the graphite. Deposition of graphite by metamorphic fluids in other geological environments has been well documented [22]. Of over 1,000 spheres and spheroids that have been examined, approximately twenty have been observed to have clearly identifiable cones on their surfaces. The cones appear highly reflective with a silver-white metallic luster. A small pyrite crystal is commonly associated with cone-bearing graphite aggregates.

The cones were studied using optical microscopy, scanning electron microscopy (SEM) (JEOL JSM-820), environmental field emission scanning electron microscopy (FESEM) (Philips XL30 FESEM), transmission electron microscopy (TEM), convergent beam electron diffraction, and Raman microspectroscopy (Renishaw 1000, $\mathrm{Ar}^{+}$laser, $514.5 \mathrm{~nm}$ excitation wavelength, $2-\mu \mathrm{m}$ spot size). Ground and polished samples were examined in reflected polarized light (Nikon Optiphot-Pol). Apex angles were measured on digital images using ImageJ software. 


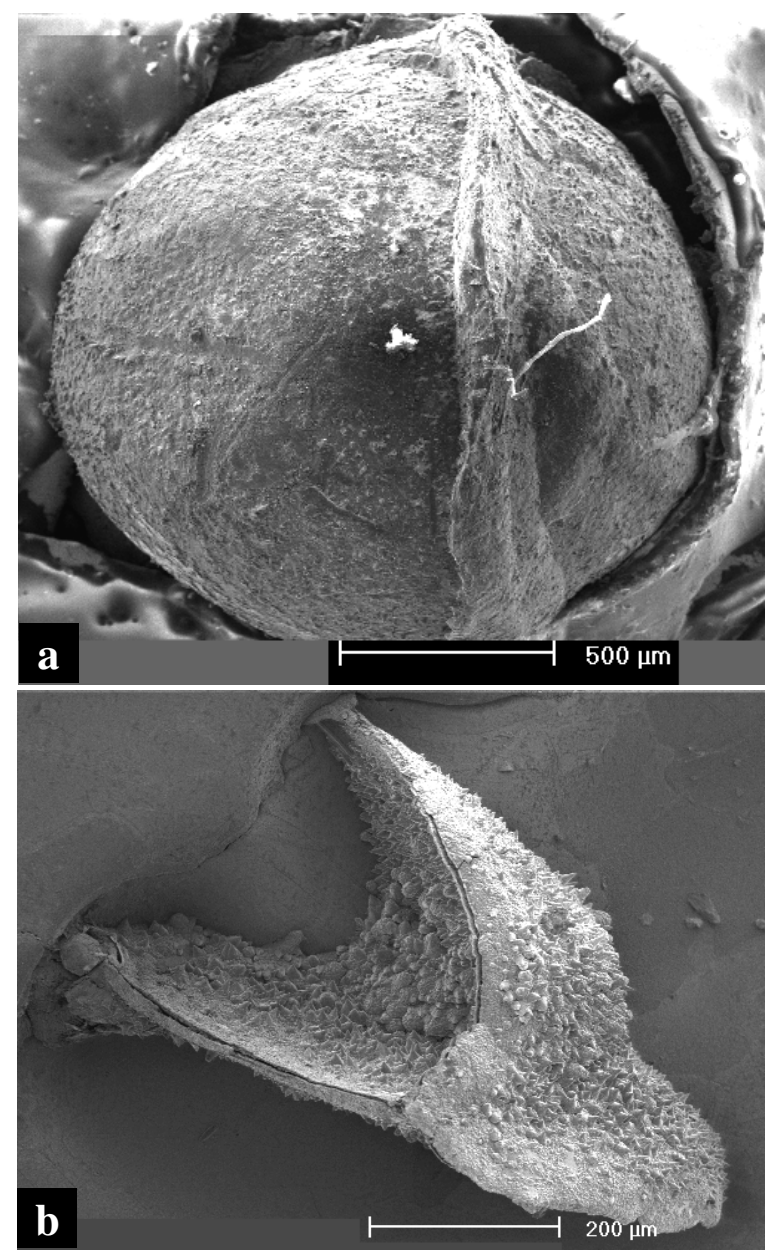

Fig. 1. (a) FESEM image of a spherical graphite aggregate with small cones on the surface (etched from calcite and mounted with copper tape). The pronounced ridge running from top to bottom is also graphite. (b) FESEM image of a cone-covered triskelial graphite aggregate partially etched from calcite. The lobes of these unusual forms seem to be correlated with calcite grain boundaries.

\section{RESTULTS AND DISCUSSION}

The microscopy study showed that cones range in size from sub-micrometer to 40 microns tall, have a variety of apex angles, and can be sharp or rounded (Figs. 1 and 2). It is striking that the cone morphologies, which are extremely rare in the mineral and material kingdom, can dominate the graphite surfaces (Fig. 2a). Moreover, all surface features, including large artichoke-like shapes (upper part of Fig. 2c) and nanoscale cones or overgrowths on large cones (Fig. 3b,c) have conical habit. This fact suggests that the growth environment rather than the substrate were responsible for the conical growth. In cross section, the cones appear to be solid. 


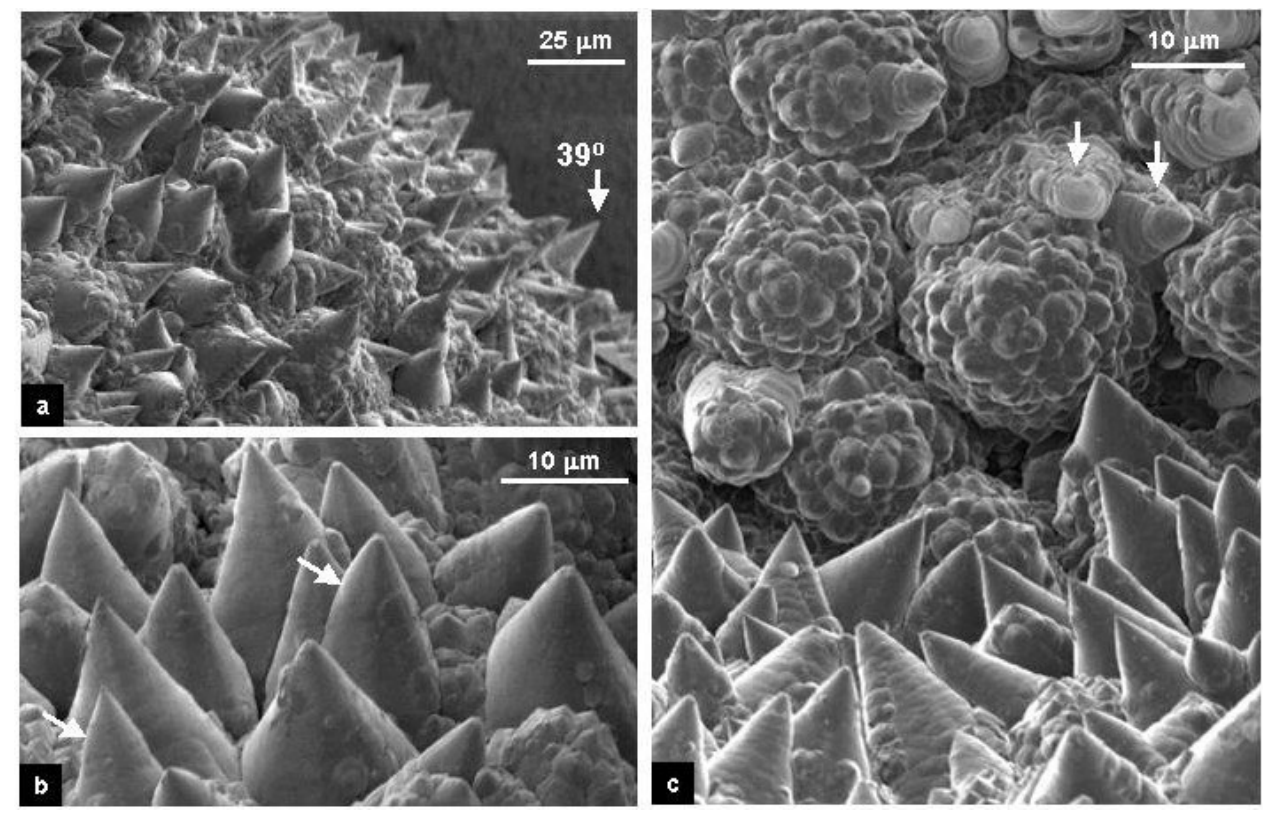

Fig. 2. FESEM images of a cone-covered graphite aggregate. (a) Low-magnification image showing complete coverage of the aggregate surface with conical structures. A $\sim 39^{\circ}$ cone is marked by an arrow. (b) Higher magnification image of the sample showing a variety of large cones with different apex angles and sharp and blunt tips. Arrows show changes in the apex angle. (c) Close up view of two surfaces which are almost perpendicular and show different cone morphologies - large cones on one surface and globular (artichoke-like) structures on the other. The latter ones are clusters of largeangle cones. Arrows show some of the cones that are ripped on the side
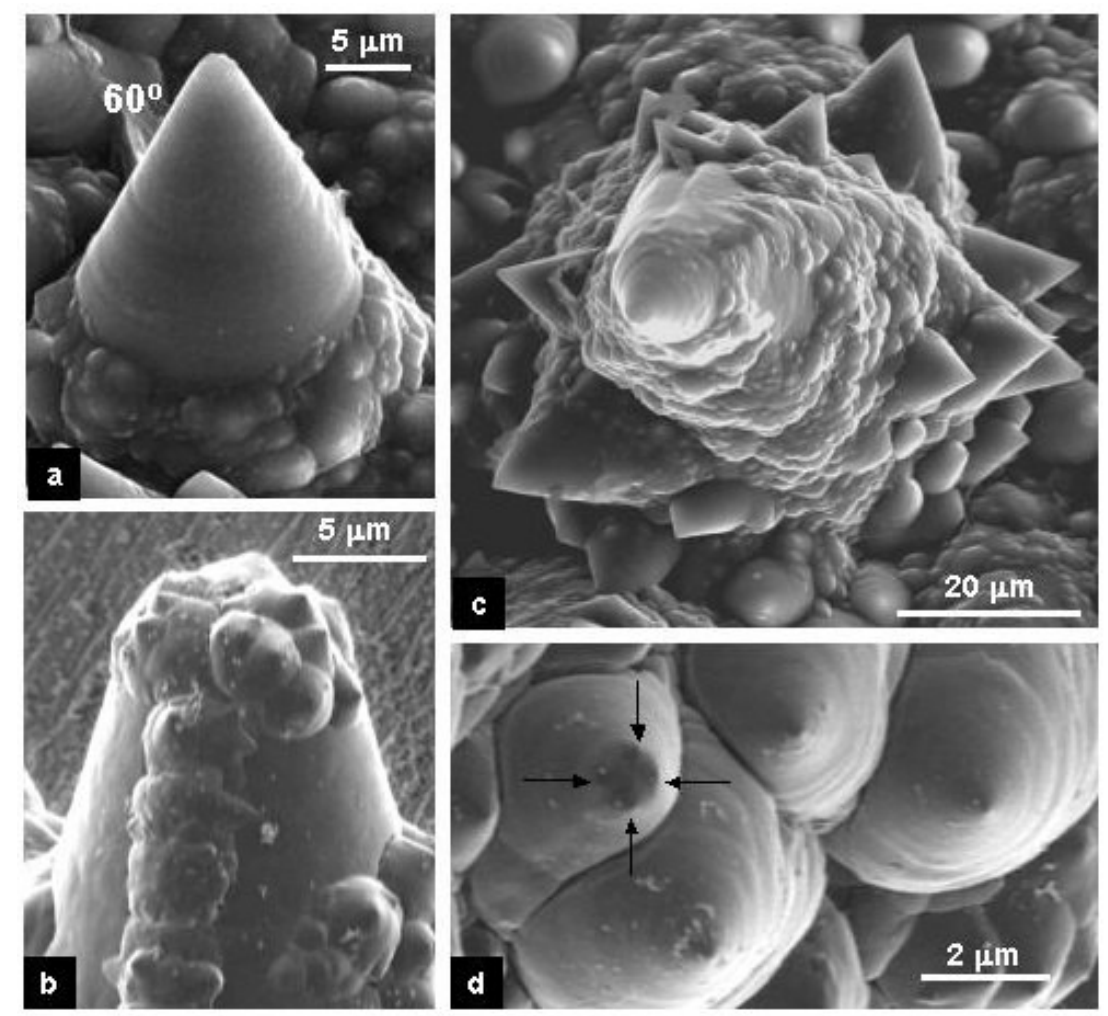

Fig. 3. Typical cone morphologies. (a) SEM image of a cone with a $60^{\circ}$ apex angle, the most common apex angle. The slightly uneven surface of the cone suggests layer growth. (b) FESEM and (c) SEM images of large cones with numerous smaller cones growing on their surface. Smaller cones covering surfaces of large cones have a broad distribution of shapes, but large apex angles prevail (c). (d) FESEM image of four cones having sharp and broad tips (multiple tips are marked by arrows). The cones are oriented to reveal their circular cross sections around the tips and layered growth (ripples). 
Apex angles of 554 cones, measured from digitized images, are presented in Fig. 4. An uncertainty of $\pm 2^{\circ}$ is estimated for most of the measurements. Uncertainties are larger, however, for many of the smaller cones, which frequently have the largest apex angles $\left(\geq 90^{\circ}\right)$. Some cones were noted to have slightly curved surfaces in cross section (marked with arrows in Fig. 2b), making a determination of the apex angle difficult. Also, the very tip of the cone may have a slightly different apex angle compared to the main part of the cone and smaller cones can grow on the tip or side walls of larger cones (Fig. 3b,c), ultimately transforming them to artichoke-shaped cones (see upper part of Fig. 2c). Additional uncertainty of the measured angles arises from the random inclination of the cones with respect to the plane of the image. For an actual apex angle $\alpha$ (Fig. 4c), the measured (projected) apex angle $\alpha^{\prime}$ as a function of the inclination angle $\phi$ of the cone with respect to its axis lying in the plane of the image is given by $\tan \left(\alpha^{\prime} / 2\right)=\tan (\alpha / 2) / \cos (\phi)$. For both positive and negative $\phi$ values, $\alpha^{\prime}$ is always greater than $\alpha$. For $|\phi| \leq 15^{\circ}, \alpha^{\prime}-\alpha$ is on the order of $2^{\circ}$ or less. Thus, only cones whose central axes were judged to lie nearly parallel to the plane of the image were measured for inclusion in Fig. 4. Approximately $3 \%$ of the cones observed have apex angles near $39^{\circ}$. These cones occur up to 45 microns tall and are up to twice as tall as the next-largest cones with other apex angles (Fig. 4a). The most common apex angle is near $60^{\circ}$ (Fig. 3a), with cones having $60^{\circ} \pm 2^{\circ}$ apex angles accounting for nearly $23 \%$ of those measured (Fig. 4a).

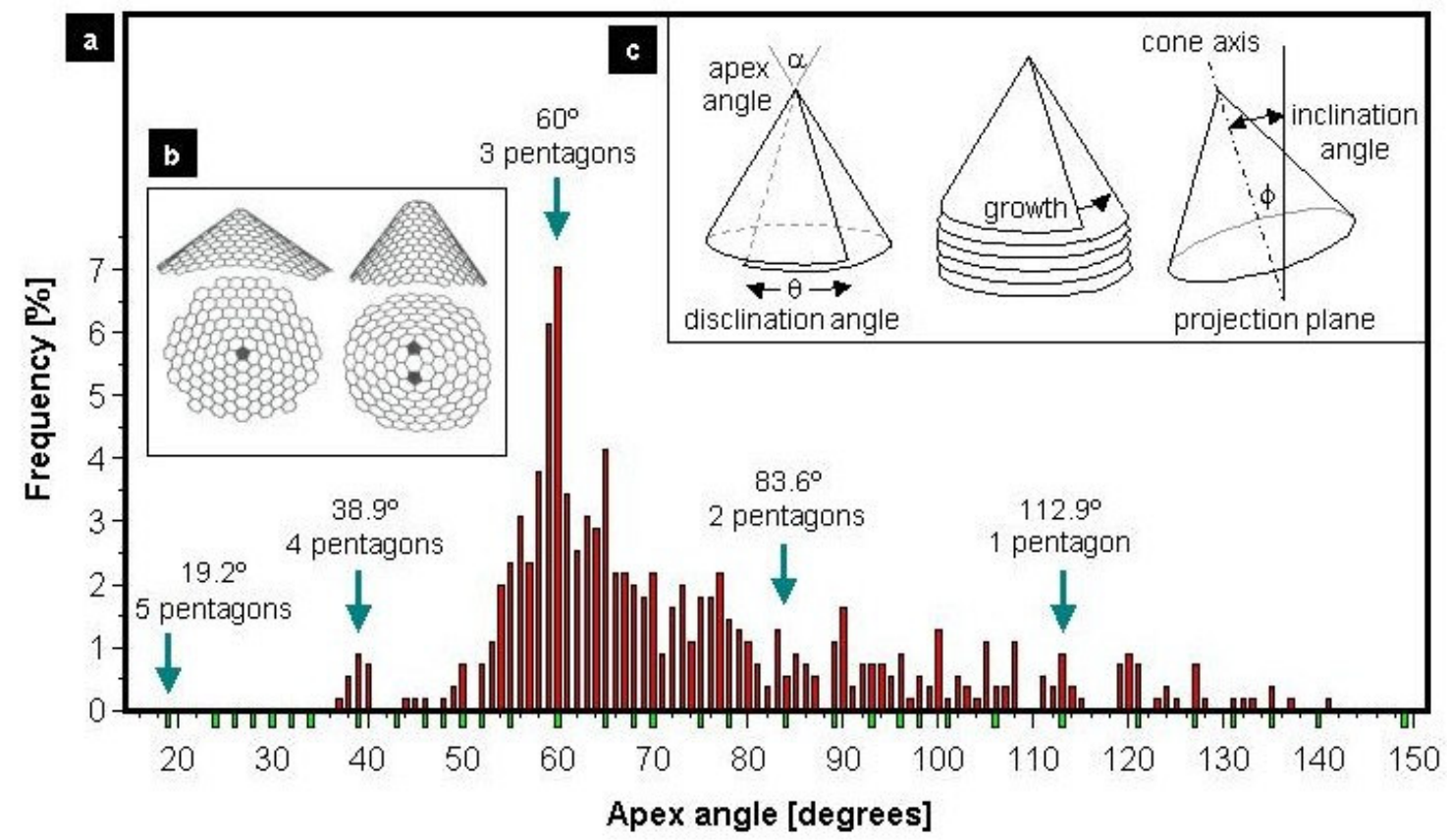

Fig. 4. (a) Histogram of the frequency of occurrence of apex angles on a single sample of graphite. Outlined bars with negative values indicate the positions of expected apex angles from the disclination model for cone-helix structures [7]. Arrows show expected apex angles for the pentagon defect model; these angles also preserve the ideal graphite stacking and are therefore expected to have relatively low energies and be more predominant. Inset (b) shows a schematic of the pentagoninduced cone formation. Inset (c) shows a schematic of cone formation by the disclination model [7].

Two mechanisms that can lead to conical shapes have been described in literature and are schematically shown in Fig. $4 \mathrm{~b}$ and $4 \mathrm{c}$. If the cone formation had been caused by pentagonal defects incorporated within graphene sheets, only a few discrete apex angles would be observed (marked by arrows in Fig. 4a). Since a broad apex-angle distribution has been found, these structures are probably based on the incorporation of a positive wedge disclination in a graphene sheet (Fig. 4c). Depending on the magnitude of the disclination, 
they can have a variety of apex angles. Double \& Hellawell [7] proposed a cone-helix model for conical graphite fibers observed by earlier workers, that fits well the observations of the natural cones of this study. The cone-helix model is based on growth around a positive disclination with a screw dislocation component. As a graphene sheet wraps around the disclination, adjacent overlapping layers are rotated with respect to one another by an angle equal to the disclination angle (Fig. 4c). Double \& Hellawell proposed that certain disclination angles, $\theta=\mathrm{n} \times 60^{\circ}$ or $\mathrm{n} \times 60^{\circ} \pm \omega$, should be energetically favorable. Here, $\mathrm{n}=0$, $1, \ldots 6$, and $\omega=13.2^{\circ}, 21.8^{\circ}, 27.8^{\circ}$ are expected low-energy (001) twist grain-boundary angles based on lattice coincidences, which are a measure of "goodness of fit" but ignore atomistic interactions and the curvature of the sheets. Thus, certain apex angles $\alpha=2 \sin ^{-1}(1$ $\theta / 360^{\circ}$ ), ranging from $6^{\circ}$ to $149^{\circ}$, should predominate over others, as shown in Fig. 4a and Table 1. Almost all of the apex angles observed in literature [3-7,11-17] (see Table 1) are consistent with the cone-helix growth model of Double \& Hellawell, except for the smallest apex angle, $\sim 3^{\circ}$, which is from cones that are probably nanotube-like scrolls $[5,23]$.

Table 1

Disclination overlap angles $(\theta)$ and corresponding cone apex angles $(\alpha)$ for laboratory-grown graphite cones.

\begin{tabular}{|c|c|c|}
\hline Overlap Angle $(\theta)$ & Apex Angle $(\alpha)$ & Reference \\
\hline $13.2^{\circ}$ & $148.9^{\circ}$ & (not observed) \\
\hline $21.8^{\circ}$ & $139.9^{\circ}$ & $7,11,12,14$ \\
\hline $27.8^{\circ}$ & $134.7^{\circ}$ & 17 \\
\hline $60^{\circ}$ & $112.9^{\circ}$ & 3,12 \\
\hline $120^{\circ}$ & $83.6^{\circ}$ & 3 \\
\hline $180^{\circ}$ & $60.0^{\circ}$ & 3,13 \\
\hline $240^{\circ}$ & $38.9^{\circ}$ & $3,14,16$ \\
\hline $300^{\circ}$ & $19.2^{\circ}$ & $3,6,16$ \\
\hline $\begin{array}{l}\text { Other combinations } \\
\left(\theta=n \times 60^{\circ} \pm \omega\right)\end{array}$ & (various) & \\
\hline $300^{\circ}+13.2^{\circ}$ & $14.9^{\circ}$ & 5 \\
\hline $300^{\circ}+27.8^{\circ}$ & $10.3^{\circ}$ & 16 \\
\hline $360^{\circ}-21.8^{\circ}$ & $6.9^{\circ}$ & 5 \\
\hline
\end{tabular}

The apex-angle distribution in Fig. 4 shows a peak near $60^{\circ}$, corresponding to three pentagons in the cone tip (Fig. 4). Consistent with the conjecture made by Double and Hellawell, cones with smaller apex angles may be disfavored because of higher elastic energy due to bending needed to form the corresponding disclinations. We have not observed any cones with apex angles smaller than approximately $36^{\circ}$ (including any at $19.2^{\circ}$ corresponding to five pentagons). On the other hand, cones with smaller apex angles have larger surface areas at their tips, compared with cones with larger apex angles, given a fixed cone diameter. This relative area factor would favor smaller apex-angle cones over larger ones during growth. These competing energetic and kinetic factors may explain the existence of the main peak in the apex-angle distribution. As noted further by Double and Hellawell, apex angles corresponding to disclinations with overlap angles equal to integer multiples of $60^{\circ}$ (marked by arrows in Fig. 4) should be energetically the most favorable, bending energy aside. Disclinations with these overlap angles preserve the graphite crystal structure without stacking faults, provided the screw component of the disclination has a Burgers vector corresponding to an even multiple of graphite's $c$-axis interplanar spacing.

Deviations in the frequency of occurrence of apex angles (Fig. 4a) from the predicted apex angles of Double and Hellawell could be due to a number of factors, including measurement errors discussed above. As suggested by the uneven surfaces of some cones (Figs. 1b,c), and the curved profile of other cones (Fig. 2b), distributions of steps on the cone surfaces could 
lead to a distribution of apex angles among cones with the same disclination overlap angle. Openings at the sides of some cones (marked with arrows in Fig. 2c) can lead to additional shape deviations. Steps on the cone surfaces (Figs. 2a,d, 5) are also an indication of a layergrowth mechanism, further suggestive of growth in a fluid rather than from solid-state transformation of carbonaceous sediments. Growth of carbon nanotubes [24], disordered conical structures [25] and planar graphite [26] from hydrothermal fluid has been observed around $700^{\circ} \mathrm{C}$. However, the significance of the calcite matrix or the commonly associated pyrite for the occurrence of cones in this work is not known.

Fig. 5. FESEM images of a $10-\mu \mathrm{m}$ tall graphite cone with distinct surface steps.

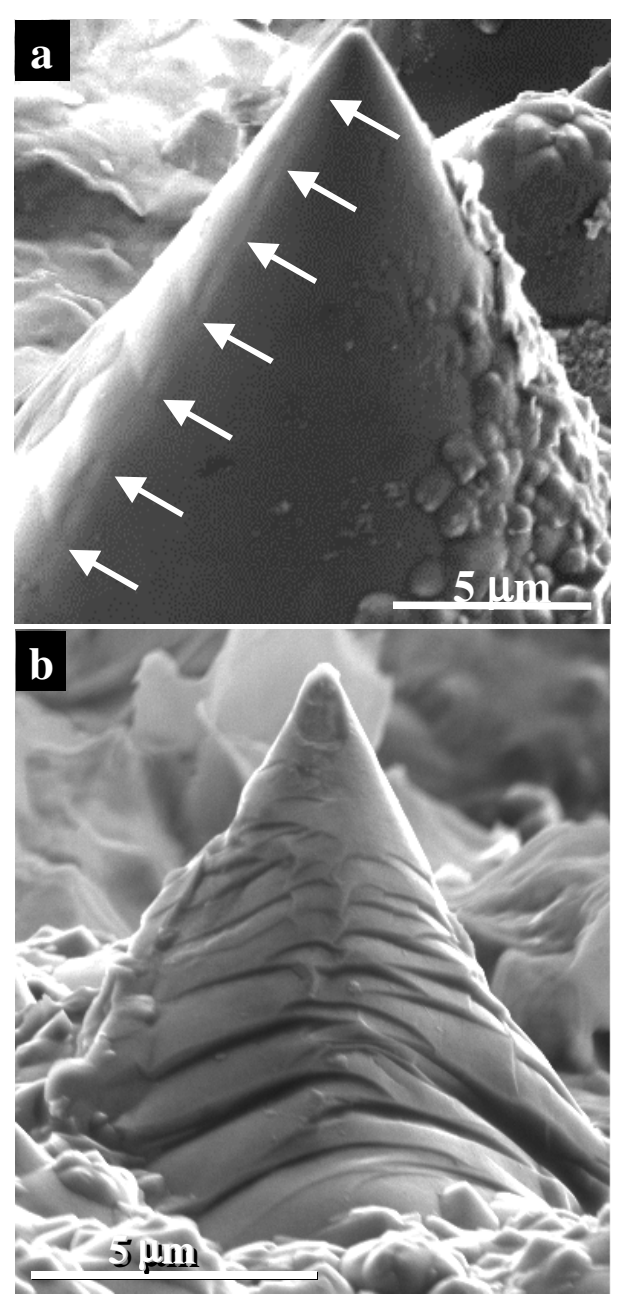

The distribution of apex angles in this study of natural graphite cones bears several similarities to that shown by Krishnan et al. [3]. In particular, they observed a large fraction of apex angles near but distinct from the five angles predicted by pentagon defects alone. Futhermore, they observed apex angles larger than $120^{\circ}$, including what appears to be the $\alpha=140^{\circ}$ apex angle resulting from zero pentagons but $\omega=21.8^{\circ}$, and which might have been expected to form based on earlier work $[7,11,12,14]$. If the cone formation had been caused solely by pentagonal defects, only a few discrete apex angles would be observed (marked with arrows in Fig. 4a) instead of a broad apex angle distribution. In addition, all cones would be very sharp. While some are (e.g., side cones in Fig. 3b), others have rounded (Fig. 3a), almost flat (Fig. 3c) or even multiple tips (Fig. 3d). Flat and rounded cone tips were shown to be formed by semi-toroidal edge termination of graphene cylinders [5]. The texture of the fracture surface of broken cones reveals curved lamellae that are consistent with the conehelix structure. Convergent beam electron diffraction patterns from a single cone on a microtomed sample are also consistent with the graphene sheets lying parallel to the cone surface. We suggest that the nucleation of pentagon defects is not the only factor determining 
their distribution of micro- and nano-cone apex angles. Cone-helix energies and kinetics might also play a role, in addition to the pentagon defect nucleation, as a mechanism explaining the distributions of apex angles of this study.

Raman microspectroscopy analysis of about 20 graphite cones (Fig. 6) showed very little variation with respect to the size or apex angle. Both, first and second order spectra were similar to that of microcrystalline graphite or multiwall nanotubes [26] with slightly upshifted $D$ and $G$ bands. Up-shift of Raman bands from the positions typical for planar graphite $\left(\sim 1355\right.$ and $1582 \mathrm{~cm}^{-1}$, respectively) may be due to the curved cone surfaces. The presence of the $D$ band in all spectra is consistent with the fact that the surfaces of the cones are not perfectly wrapped in a continuous graphene sheet, but rather that edges of graphene layers form growth steps as seen in Figs. $2 \mathrm{~d}$ and 5. Broadening of the $G$ band $\left(\sim 34 \mathrm{~cm}^{-1}\right.$ in cones compared to $14 \mathrm{~cm}^{-1}$ for a planar single crystal) can also result from the conical wrapping of the layers and bending the graphene sheets. The intensity of $D$ band relative to $G$ band was lower compared to carbon whiskers [17], which are assumed to be formed by the same disclination mechanism as the cones, but higher than in graphite polyhedral crystals (GPC) [4]. The second order spectrum of the cones was also much weaker than that of GPCs or whiskers.

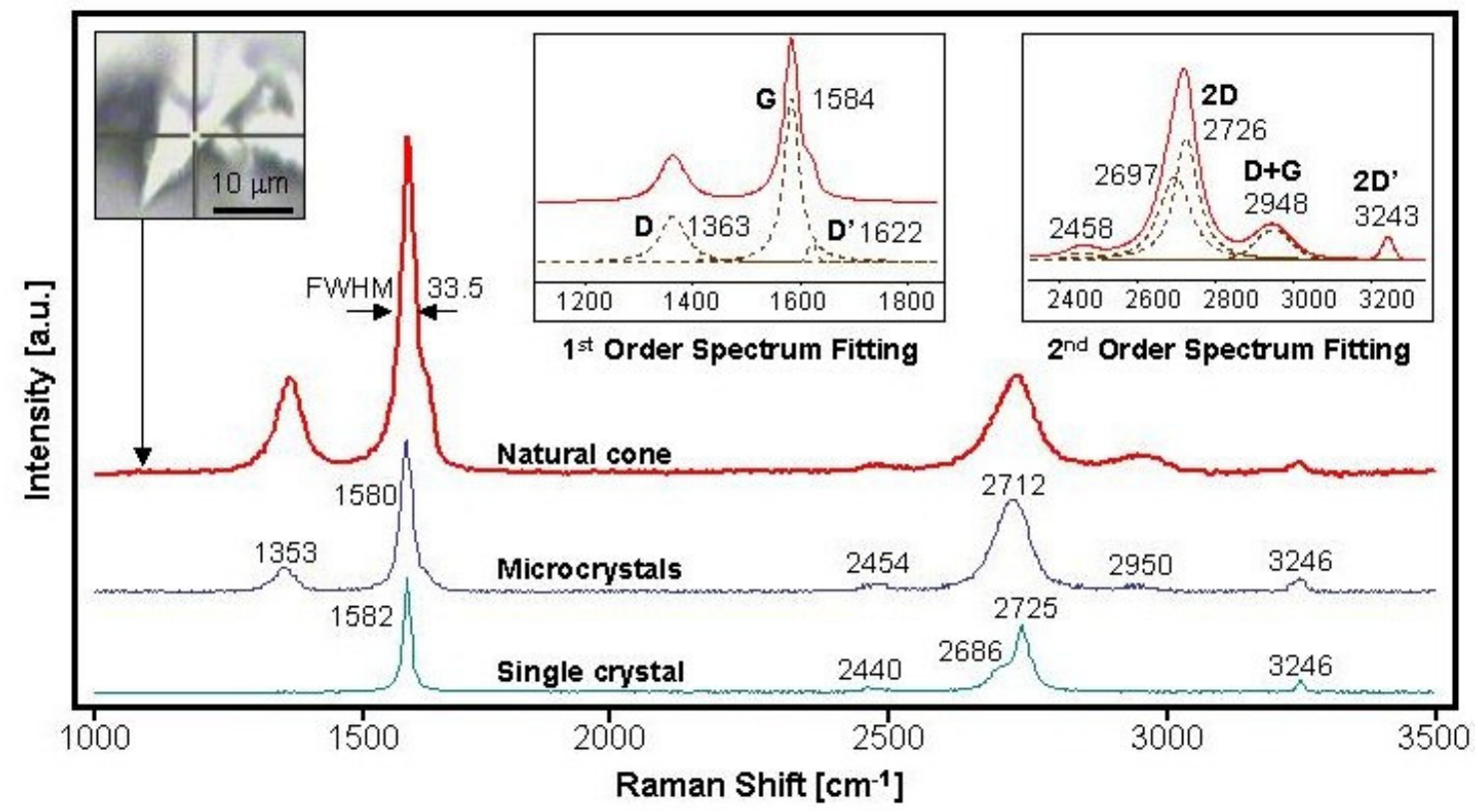

Fig. 6. Raman spectra of a graphite cone in comparison with microcrystalline graphite and a planar graphite single crystal. Insets show deconvolved regions of the first and second order spectra from the cone. Cross-hair in the optical image indicates the position of the Raman probe.

\section{CONCLUSIONS}

The cone-helix model [7] is successful in predicting the expected apex angles of graphite cones synthesized under various laboratory conditions and formed naturally from fluids during metamorphism. The consistency of the model with observed apex-angle distributions suggests that the nucleation of pentagon defects is not the only factor in determining graphite cone morphologies, but that the energetics of layer-layer interactions between the graphene sheets also plays an important role in determining micro- and nano-scale carbon structures. A better understanding of the nucleation and growth mechanisms of these systems could help increase the yields of nanotubes or nanocones relative to other carbon structures in the laboratory synthesis. Furthermore, the occurrence of fluid-grown micron-sized graphite cones, particularly those with apex angles corresponding to disclination overlap angles that preserve the ideal ...ABAB .. stacking, suggests that it might be possible to grow macroscopic arrays of nano- and micro-sized graphite cones without co-production of other graphite structures. 
Acknowledgements- We are grateful to Jeffrey G. Meyer for supplying bulk samples from which the cones of this study were discovered. We also thank Robert Hudyma for supplying bulk material for study. We thank John Rakovan and Mark Krekeler for preparing microtomed samples, Stephen Hackney for TEM work and Nevin Naguib for experimental assistance. Work done at Drexel University was supported by the US Departmnent of Energy.

\section{REFERENCES}

[1] Bernaerts D, Amelinckx S, Tendeloo GV, Landuyt JV. Electron microscopy of carbon nanotubules and related structures. J Phys Chem Solids 1997; 58:1807-1813.

[2] Bourgeois L, Bando Y, Kurashima K, Sato T. Co-produced carbon and boron nitride helical cones and the nucleation of curved BN sheets. Phil Mag A 2000; 80:129-142.

[3] Krishnan A, Dujardin E, Treacy MMJ, Hugdahl J, Lynum S, Ebbesen TW. Graphitic cones and the nucleation of curved carbon surfaces. Nature (London) 1997; 388:451-454.

[4] Gogotsi Y, Libera JA, Kalashnikov N, Yoshimura M. Graphite polyhedral crystals. Science 2000; 290:317-320.

[5] Dimovski S, Libera JA, Gogotsi Y. A novel class of carbon nanocones. Mat Res Soc Symp Proc 2002; 706:Z6.27.1-Z6.27.6.

[6] Ge M, Sattler K. Observation of fullerene cones. Chem Phys Lett 1994; 220:192-196.

[7] Double DD, Hellawell A. Cone-helix growth forms of graphite. Acta Metall 1974; 22:481-487.

[8] Iijima S, Yudasaka M, Yamada R, Bandow S, Suenaga K, Kokai F, Takahashi, K. Nanoaggregates of single-walled graphitic carbon nano-horns. Chem Phys Lett 1999; 309:165-170.

[9] Harris PJF. Carbon nanotubes and related structures. Cambridge (UK): Cambridge Univ Press. 1999.

[10] Jaszczak JA. Graphite: Flat, fibrous and spherical. In: Mendenhall GD, Greenberg A, Liebman JF, editors. Mesomolecules: From molecules to materials. New York: Chapman \& Hall, 1995:161-180.

[11] Tsuzuku T. Dislocations in graphite crystals. In: Proceedings of the Third Conference on Carbon. New York: Pergamon, 1957:433-450.

[12] Gillot J, Bollman W, Lux B. Cigar shaped graphite crystals with a conical structure. Carbon 1968; 6:381-387.

[13] Lieberman ML, Mills CR, Miglionico CJ. Growth of graphite filaments. Carbon 1971; 9:633635.

[14] Haanstra HB, Knippenberg WF, Verspui G. Columnar growth of carbon. J Cryst Growth 1972; 16:71-79.

[15] Amelinckx S, Luyten W, Krekels T, Van Tendeloo G, Van Landuyt J. Conical, helically wound, graphite whiskers: A limiting member of the "fullerenes"? J Cryst Growth 1992; 121:543-558.

[16] Muradov N, Schwitter A. Formation of conical carbon structures on vapor-growth carbon filaments. Nano Letters 2002; 2:673-676.

[17] Dong J, Shen W. Tatarchuk B. Origin of strong G' band in Raman spectra of carbon whiskers. Appl Phys Lett 2002; 80:3733-3735.

[18] Jaszczak JA, Robinson GW. Spherical and triskelial graphite from Gooderham, Ontario, Canada. Rocks \& Minerals 2000; 75:172-173.

[19] Van der Pluijm BA, Carlson KA. Extension in the Central Metasedimentary Belt of the Ontario Grenville: Timing and tectonic significance. Geology 1989; 17:161-164.

[20] Carlson KA, Van der Pluijm BA, Hanmer S. Marble mylonites of the Bancroft shear zone; evidence for extension in the Canadian Grenville. Geol Soc Am Bull 1990; 102:174-181.

[21] Weis PL. Graphite skeleton crystals- A newly recognized morphology of crystalline carbon in metasedimentary rocks. Geology 1980; 8:296-927

[22] Luque FJ, Pasteris JD, Wopenka B, Rodas M, Barrenechea, JF. Natural fluid-deposited graphite: Mineralogical characteristics and genesis. Am J Sci 1998; 298:471-498.

[23] Gogotsi Y, Dimovski, S, Libera JA. Conical crystals of graphite. Carbon 2002; 40:2263-2267.

[24] Gogotsi Y, Libera J, Yoshimura M. Hydrothermal synthesis of multiwall carbon nanotubes. J Mater Res 2000; 15:2591-2594.

[25] Gogotsi YG, Nickel, KG. Formation of filamentous carbon from paraformaldehyde under high temperatures and pressures. Carbon 1998; 36:937-942.

[26] Libera J, Gogotsi Y. Hydrothermal synthesis of graphite tubes using Ni catalyst. Carbon 2001; 39:1307-1318. 